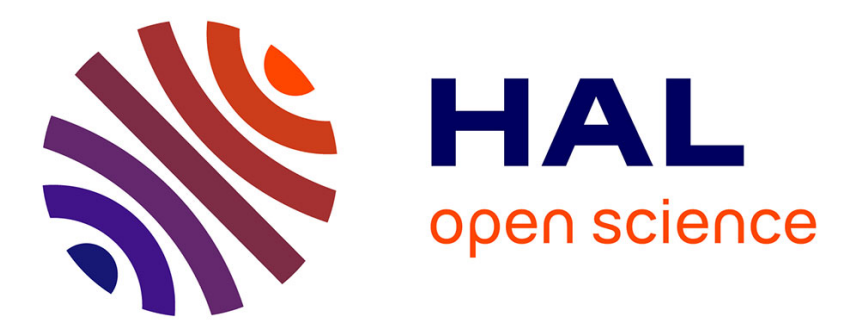

\title{
Acceptabilité et faisabilité de la psychothérapie par les patients avec trouble de personnalité limite
}

L. Cailhol, S. Bouchard, A. Belkadi, G. Benkirane, G. Corduan, S. Dupouy, E. Villeneuve, J.-D. Guelfi

\section{- To cite this version:}

L. Cailhol, S. Bouchard, A. Belkadi, G. Benkirane, G. Corduan, et al.. Acceptabilité et faisabilité de la psychothérapie par les patients avec trouble de personnalité limite. Annales Médico-Psychologiques, Revue Psychiatrique, 2010, 168 (6), pp.435. 10.1016/j.amp.2009.06.020 . hal-00659507

\section{HAL Id: hal-00659507 https://hal.science/hal-00659507}

Submitted on 13 Jan 2012

HAL is a multi-disciplinary open access archive for the deposit and dissemination of scientific research documents, whether they are published or not. The documents may come from teaching and research institutions in France or abroad, or from public or private research centers.
L'archive ouverte pluridisciplinaire HAL, est destinée au dépôt et à la diffusion de documents scientifiques de niveau recherche, publiés ou non, émanant des établissements d'enseignement et de recherche français ou étrangers, des laboratoires publics ou privés. 


\section{Accepted Manuscript}

Title: Acceptabilité et faisabilité de la psychothérapie par les patients avec trouble de personnalité limite

Authors: L. Cailhol, S. Bouchard, A. Belkadi, G. Benkirane, G. Corduan, S. Dupouy, E. Villeneuve, J.-D. Guelfi

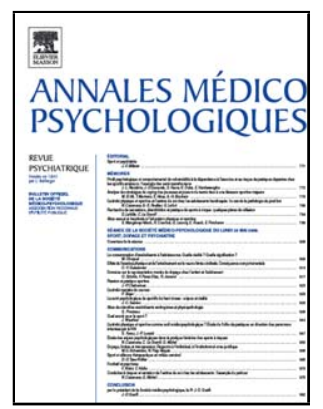

PII: $\quad$ S0003-4487(10)00140-X

DOI: $\quad$ doi:10.1016/j.amp.2009.06.020

Reference: $\quad$ AMEPSY 1159

To appear in: $\quad$ Annales Médico-Psychologiques

Received date: $\quad 12-12-2008$

Accepted date: $\quad$ 27-1-2009

Please cite this article as: Cailhol L, Bouchard S, Belkadi A, Benkirane G, Corduan G, Dupouy S, Villeneuve E, Guelfi J-D, Acceptabilité et faisabilité de la psychothérapie par les patients avec trouble de personnalité limite, Annales medio-psychologiques (2008), doi:10.1016/j.amp.2009.06.020

This is a PDF file of an unedited manuscript that has been accepted for publication. As a service to our customers we are providing this early version of the manuscript. The manuscript will undergo copyediting, typesetting, and review of the resulting proof before it is published in its final form. Please note that during the production process errors may be discovered which could affect the content, and all legal disclaimers that apply to the journal pertain. 


\section{Mémoire}

Acceptabilité et faisabilité de la psychothérapie par les patients avec trouble de personnalité limite

Acceptability and feasibility of psychotherapy for patients with borderline personality disorder

L. Cailhol $^{\text {a,b }}$, S. Bouchard ${ }^{\text {c }}$, A. Belkadi ${ }^{\text {d }}$, G. Benkirane ${ }^{\text {d }}$, G. Corduan ${ }^{\text {d }}$, S. Dupouy ${ }^{\text {d }}$, E. Villeneuve ${ }^{c}, J^{-D}$. Guelfi ${ }^{\text {e }}$

${ }^{a}$ INSERM CIC9302 Toulouse, CHU Toulouse, Hôpital Purpan, France

${ }^{b}$ Centre Hospitalier Général, Pôle Urgences, UF psychiatrie, 100, rue Léon Cladel, 82000 Montauban, France

${ }^{c}$ Institut universitaire en santé Mentale de Québec, Centre de traitement Le faubourg SaintJean, Programme-clientèle des troubles sévères de la personnalité, Institut universitaire en santé mentale de Québec, 175 rue Saint-Jean, Québec, (Qc) G1R 1N4, Canada

${ }^{d}$ Service de psychiatrie et de psychologie médicale, CHU Casselardit, 170, avenue de Casselardit, TSA 40031, 31059 Toulouse Cedex, France

${ }^{e}$ Clinique des Maladies Mentales et de l'Encéphale, Service du Pr Rouillon, Hôpital SainteAnne, 100, rue de la Santé, 75674 Paris Cedex 14, France

Auteur correspondant: Lionel Cailhol, Centre Hospitalier Général, UF Urgences Psychiatriques - Pôle Urgences, 100, rue Léon Cladel, 82000 Montauban, France Tél. : 0563928282

Adresse email : 1.cailhol@ch-montauban.fr

Texte reçu le 12 décembre 2008 ; accepté le 27 janvier 2009

Cet article a fait l'objet d'une communication à la troisième édition du colloque Troubles de la personnalité : gestion de crises et alternatives à la psychothérapie. Montréal, (Québec), 22 et 23 mai 2008.

\section{Résumé}

Le trouble de personnalité limite est un trouble grave associé à de sérieuses limitations psychosociales. La prévalence de ce trouble est de 1,8\%. Les perspectives de santé publique convergent avec les observations cliniques pour questionner leurs trajectoires de soin. En 
effet, les psychothérapies sont peu mises en œuvre pour des raisons de moyens et d'acceptabilité par les sujets eux-mêmes. À travers une revue de littérature, nous présentons des données sur l'accessibilité et l'utilisation des services de soins de cette population. L'efficacité des approches psychothérapeutiques, considérées comme le gold-standard thérapeutique, seront considérées à l'aune de leurs limites. Enfin, nous proposerons des alternatives à ces approches, issues de la littérature médicale et d'un exemple québécois d'organisation multidisciplinaire de soins. En conclusion, l'hétérogénéité des situations cliniques regroupées dans le trouble de personnalité limite se confronte aux limites d'une approche dichotomique (psychothérapie structurée ou parcours de soin chaotique), se manifestant par une acceptabilité réduite des prises en charge actuelles. Ce constat incite à repenser la cohérence de l'offre de service faite à cette patientèle sévère.

Mots clés : Psychothérapie ; Réseau de soin ; Trouble de personnalité borderline 


\section{Abstract}

Background - Borderline Personality Disorder (BPD) is a pervasive patern of instability and impulsivity associated with serious psychosocial limitations and a significant risk of suicide (4-10\%). The prevalence of this disorder is around $1.8 \%$ in the general population. In a public health perspective, the high financial cost arising from this disorder (around 17.000€ per year) requires optimizing their care utilization carefully. This medicoeconomic aspect converges with clinical observations of instability in the health network ; BPD patients meet at least 7 psychiatrists. Indeed, psychotherapy are less accessible, particularly in some geographic area.

Objective - Discuss strenght and weakness of psychotherapy for BPD in the real world

Method - Through a a non systematic literature review, we present data on the availability and use of health services by people with BPD.

Results - The effectiveness and limitations of psychotherapeutic approaches, considered as the gold-standard therapy, are reviewed. All the psychotherapies assessed by Randomised Control Trials seems to be effective. However not all the psychotherapies have the same level of evidence for core symptoms of BPD. Furthermore both the length of most of the psychotherapies suggested to these unstable patients and the number of participants (Dialectic Behavioral Therapy and Mentalization Based Treatment) needed to constitute the team for some of them are serious limitations. Moreover, even available, as shown by observational studies, high drop-out in studies they have a limited acceptability for BPD themselves. Some of them have less drop-out (Dialectic Behavioral Therapy) or a better therapeutic alliance (Schema Focused Psychotherapy) than other one. A presentation of alternatives to psychological approaches is proposed, particularly an example of a Quebec multidisciplinary care organization covering the range of service needs of people with BPD.

Conclusions - The heterogeneity of clinical situations grouped in the BPD, is confronted with the limits of a dichotomous approach (structured psychotherapy or chaotic utilization of care), manifested by a reduced acceptability of care today. This encourages them to rethink the consistency of the service offer to these severe patients.

Keywords: Borderline personality disorder; Community network; Psychotherapy 


\section{Introduction}

Le trouble de la personnalité limite (TPL) est une entité clinique caractérisée par l'instabilité relationnelle et identitaire, l'impulsivité et la dysrégulation émotionnelle [1]. Au plan épidémiologique, le TPL est fréquent avec une prévalence de 1,8\% de la population générale [42], il est associé à de graves limitations psychosociales (instabilité au travail, compétences parentales limitées, instabilités relationnelles...) et à un risque important de suicide, estimé entre $4 \%$ et $10 \%$ [28]. À la fréquence et la gravité clinique du TPL sont associés des coûts financiers substantiels pour la société, d'environ 17000 euros par an [3].

Face à la sévérité et à la complexité du TPL, les sociétés savantes et les systèmes sanitaires proposent des solutions. Pour les premières, s'appuyant sur l'evidence-based medecine, elles soutiennent la priorisation de la psychothérapie [1]. Les traitements pharmacologiques sont quant à eux considérés comme actifs sur des cibles spécifiques [9,38]. Pour les seconds, la France s'appuie sur l'organisation sectorielle et son articulation avec la psychiatrie libérale. D'autres zones sanitaires, tels que la Suisse romande et le Québec pour ce qui est de la francophonie, associent à ces prises en charge de $2^{\mathrm{e}}$ ligne des centres de soins spécialisés pour les Troubles Graves de la Personnalité, dont les TPL.

Pourtant, dans ces régions comme en France, la pratique clinique est confrontée à la difficulté d'accès à une psychothérapie de long terme, pour une part importante de nos patients, alors qu'elles sont recommandées. Cette constatation, étayée par les données d'épidémiologie clinique, nous dicte d'entamer une réflexion sur les alternatives à la psychothérapie. Des propositions de soins novatrices doivent s'articuler aux diversités des réseaux de soins, en vue d'offrir un éventail de services plus cohérent et plus sensible aux particularités de cette problématique complexe.

Notre article a pour objectif de discuter les limites d'une approche strictement psychothérapique de la prise en charge des patients avec TPL, en proposant des alternatives innovantes utilisables dans nos systèmes sanitaires. 


\section{Accès et utilisation des services de soins par les patients présentant un TPL}

\subsection{Utilisation et navigation dans les soins}

Les patients avec TPL demeurent des utilisateurs important des soins de santé. En effet, les données épidémiologiques concernant la prise en charge de ces patients nous enseignent qu'ils utilisent davantage d'hospitalisation, de psychothérapie, de suivi psychiatrique, de passages aux urgences, que les patients déprimés ou présentant d'autres troubles de personnalité $[11,47]$. Cette importante utilisation est associée à de nombreux changements de prise en charge (sept psychiatres en moyenne par exemple). Ces constatations, largement reconnues par les cliniciens et les études prospectives, ne permettent néanmoins pas de conclure quant à l'adéquation entre les besoins et les soins fournis. Par ailleurs, peu de facteurs ont été étudiés pour expliquer cette importante utilisation [22].

À l'instar de P. Fallon, il serait sûrement utile de distinguer, d'un côté les patients navigants dans le système de soins de façon constructive et cohérente, comme si les changements de prise en charge se coordonnaient rationnellement, et, de l'autre, nous retrouverions des patients en perpétuelle mouvance, utilisant le système de soins de façon telle qu'elle manifestait le chaos interne du sujet [24]. Le premier cas correspond à une utilisation importante, progressive et adaptée au stade de changement du patient: hospitalisations brèves, fréquentation d'hôpital de jour, suivi pharmacologique, et finalement psychothérapie de long terme. On y constate une courbe positive d'apprentissage et une réduction progressive des symptômes aigus associés au TPL. Dans le deuxième cas, le profil correspond à une utilisation des services de soins marquée par l'instabilité, peu coordonnée, tels que des hospitalisations itératives dans différents établissements, de nombreux changements de thérapeutes et une absence de responsable de dossier. Ces parcours de soins seraient peu ou pas corrélés avec l'apprentissage d'une meilleure gestion du trouble par la personne atteinte.

\section{2. Éclairage clinique}

L'implication du patient dans une démarche structurée n'est possible que si la relation thérapeutique échappe pour une large part à l'instabilité pourtant caractéristique du TPL. Cette proposition de soins ne peut donc apparemment concerner qu'un sous-groupe particulier de patients sélectionnés, jugés « amenables » à la psychothérapie [40,41]. 
De plus, le morcellement identitaire et la désorganisation du fonctionnement de la personne présentant un TPL se confrontent de façon peu efficiente aux structurations des traitements proposés et aux rigidités des systèmes de soins.

Par ailleurs, si l'on considère que l'alliance thérapeutique se définit notamment par un accord sur les buts et les tâches [14], le défi consiste à explorer et choisir avec le sujet des objectifs de traitement clairs. L'instabilité de l'identité complique ce choix d'objectifs qui généralement devient tout aussi fluctuant dans le temps et selon les situations. Le flou et la versatilité de la demande du patient, ainsi que le caractère d'urgence soutenue par l'impulsivité, représentent vraisemblablement des obstacles à une démarche psychothérapique.

À ces caractéristiques cliniques inhérentes au TPL s'associent de nombreuses cooccurrences avec d'autres troubles de personnalité ou d'autres troubles cliniques, dépression, anxiété et addictions, complexifiant d'autant le rationnel de prise en charge clinique [19,27]. Dans cette diversité clinique, l'entrée dans les soins se fait souvent à l'occasion d'une recrudescence symptomatique d'anxiété ou de dépression, ou de troubles du comportement telles que des conduites suicidaires. Le risque, aussi bien pour les patients que pour les médecins, est de se limiter à cette façade symptomatique que l'on cherche à réduire, sans chercher à atteindre le problème de fond constitué par la structure de la personnalité.

\subsection{Du côté des soignants et des systèmes sanitaires}

Tout d'abord, le paradoxe de l'offre de soins aux personnes présentant un TPL consiste à proposer à un sujet instable et impulsif un projet de psychothérapie long terme nécessitant persistance et autocontrôle. Cette offre s'inscrit dans un monde idéal où ces personnes atteintes font une démarche autonome, pour recourir à des soins appropriés à leur condition. Cependant, ce ne sont pas toutes ces personnes qui iront ou seront dirigées vers des dispositifs de soins. Nous pouvons estimer qu'une portion significative de ces personnes requerront des soins, mais pas nécessairement dans les lieux ad hoc (urgence, unités d'hospitalisation, consultations). Par exemple, compte tenu de comportements antisociaux, certains se retrouvent dans des parcours judiciaires (détention, injonction). Pour d'autres, leurs difficultés identitaires, affectives, et leur impulsivité favorisent leur désinsertion et les dialogues s'amorcent prioritairement avec les acteurs sociaux.

De plus, même lorsque les services sont disponibles et que le patient paraît motivé à recevoir de l'aide, il semble que dans plusieurs cas, celui-ci ne soit pas efficacement dirigé 
dans les services spécialisés de traitement du trouble. Les données provenant de certaines populations cliniques mettent en valeur la faible accessibilité de ces patients aux soins de santé mentale. À titre d'exemple, les données issues des urgences de l'hôpital de Genève, à partir d'une cohorte de patients admis pour intoxication médicamenteuse volontaire, montrent que ces patients sont repérés, qu'ils présentent des critères de gravité, mais qu'ils ne sont pas orientés différemment des autres patients [20]. En particulier, ils n'accèdent pas aux programmes de soins qui leur sont dédiés dans la ville. D'autre part, les données issues de la médecine générale, aux États-Unis [26] ou en France [37], soulignent que ces patients ne sont souvent pas repérés et bénéficient de peu d'accès aux soins de santé mentale.

Ces données ne permettent pas de savoir si cette faible accessibilité est liée à la désorganisation mentale et sociale des patients présentant un TPL ou à une certaine désorganisation structurale du réseau de soins face aux défis que représente le traitement multimodal du TPL. Quoi qu'il en soit, une combinaison de facteurs concourt à faire de la prise en charge par le système de soins du TPL un problème de santé publique de taille, et plus encore si l'on tient compte de la réalité des autres troubles sévères de personnalité. Il reste énormément à faire pour que ce trouble reçoive une attention des autorités à la hauteur de ce que l'on a fait pour d'autres troubles, notamment les maladies affectives et la schizophrénie.

De plus, leur aptitude limitée dans la gestion des stress interpersonnels, dont le contretransfert négatif des thérapeutes, peut entraver considérablement leur capacité rationnelle à adhérer à un plan de soins adapté [46]. En effet, l'implication émotionnelle du thérapeute est souvent vive. C'est pourquoi la ligne est souvent mince entre le maintien à tout prix de l'alliance (surimplication) et l'abandon du travail thérapeutique [10]. Or, la nécessité de l'établissement d'une bonne alliance thérapeutique est à la fois une condition difficile à atteindre et une variable nécessaire à la psychothérapie [39]. Les manifestations d'une pauvre alliance thérapeutique sont de plusieurs natures: exacerbation de la symptomatologie, absences répétées en traitement, voire abandon définitif. En effet, entre $20 \%$ et $40 \%$ des patients abandonnent leur psychothérapie avant la fin de la première année d'un traitement devant pourtant durer de trois à cinq ans selon les auteurs [5].

\section{Efficacité et limites du projet de psychothérapie}

\subsection{Efficacité des psychothérapies}


Malgré la complexité et la sévérité des problèmes associés au TPL, il demeure qu'une portion significative de patients peuvent maintenant profiter de traitements avérés efficaces. Les données de la littérature amènent un faisceau convergent d'études soutenant en général l'efficacité des psychothérapies de long terme dans la prise en charge des TPL [5,13]. En particulier, elles ont une action sur les symptômes comportementaux tels l'autodestruction, l'hostilité et les tentatives de suicide, sur les symptômes affectifs, sur l'intensité des caractéristiques du trouble lui-même (par exemple l'impulsivité) et sur la persistance en traitement. Ces traitements psychothérapeutiques, d'inspiration psychanalytique ou cognitivocomportementale, ne paraissent pas apporter les mêmes résultats sur ces différentes dimensions $[25,36]$.

\subsection{Motivation au changement}

Peu d'études fournissent des données sur les éléments de motivation des patients présentant un TPL à entamer une démarche psychothérapique. La seule étude empirique disponible de van Beek et Verheul (2008) [8] suggère que la motivation de ces patients serait constituée de deux dimensions: le besoin d'aide (expliquant 39\% de la variance de motivation) et le degré de préparation à changer (17\% de la variance). Ces auteurs soulignent que la motivation au traitement de ces patients est fortement associée au niveau de détresse psychologique éprouvée par la personne. L'expérience clinique suggère que le besoin d'aide est le plus souvent associé à une demande de solutions rapides. Les demandes sont centrées sur des problèmes psychosociaux ponctuels, comme une rupture sentimentale ou des difficultés financières, et non sur le problème de personnalité. De plus, l'instabilité de la motivation et l'ambivalence face au besoin de changement rendent l'offre de soins à long terme sans intérêt, voire inappropriée pour ce type de patient. Ce contexte suggère la présence d'un hiatus entre la demande affichée des patients et les cibles des psychothérapies validées dans la littérature internationale.

\subsection{Acceptabilité des traitements}

À ces difficultés portant sur l'acceptation d'une démarche psychothérapique par le patient doivent être ajoutés les taux d'attritions élevés rapportés par les études cliniques. Ces taux varient de $12 \%$ [6] à $40 \%$ [25] selon le mode de psychothérapie étudié, et sont sensiblement supérieurs à ceux retrouvés dans les études sur la dépression qui sont de $6 \%$ à 
$20 \%$. Sur ce point, les différentes approches de traitement pour le TPL ne semblent pas avoir les mêmes taux de rétention. La Mentalization Based Therapy (MBT) [4,6,7], fondée sur une approche psychodynamique, et la Dialectic Behavioral Therapy (DBT) [15,29-32,43], d'inspiration comportementale et bouddhiste, fournissent les taux d'attrition les plus bas. La Transference Focused Psychotherapy (TFP) [21,25] reposant sur une compréhension psychanalytique et l'interprétation du transfert, apporte les taux les plus élevés d'attrition. Dans cette thérapie, les scores d'alliance thérapeutique semblent plus bas que dans la Schema Focused Therapy (SFT), une approche cognitive validée de traitement du TPL [25,39]. De ces données, nous retenons que l'acceptation du traitement psychothérapique diffère selon les modèles utilisés. Outre la qualité de l'alliance, plusieurs facteurs sont évoqués pour expliquer ces différences : sévérité de la pathologie, mauvais appariement approche/patient, ambition des objectifs de traitement, importance donnée à la neutralité thérapeutique, et degré de soutien émotionnel.

\subsection{Faisabilité des traitements psychosociaux}

Enfin, les principaux modèles psychothérapiques du TPL disposant aujourd'hui de données probantes, demandent tous des moyens humains très importants et coûteux. La DBT de Linehan, la TFP de Kernberg, la SFT de Young et la MBT de Fonagy nécessitent tous la mise en place d'une thérapie individuelle hebdomadaire ou bihebdomadaire et d'une supervision spécialisée de l'équipe de traitement. La MBT et la DBT ajoutent à ces services individuels une approche de groupe constituée de séances d'une durée d'environ deux heures par semaine et un dispositif de soutien entre les séances. Avec une telle intensité de traitement de deux à cinq heures par semaine et pour une durée de un à cinq ans de traitement, il est difficilement envisageable que des centres de traitements publics puissent offrir de tels services en dehors des grandes villes du pays.

\section{Alternatives à la psychothérapie}

Dans un contexte de ressources limitées et face à la faible acceptabilité des traitements optimaux, la communauté clinique doit considérer les alternatives à la psychothérapie pour les patients présentant un TPL ainsi que d'autres troubles sévères de personnalité.

Plusieurs voies sont possibles. De nouveaux programmes brefs et/ou de groupe permettent d'envisager des approches alternatives ou adjonctives plus faisables, notamment 
dans des zones sanitaires n'ayant pas les moyens de mettre en auvre des programmes spécialisés $[14,33,34,45]$. Il est à noter aussi que les programmes de crise et les hôpitaux de jour bénéficient de l'avantage d'être utilisables pour différentes pathologies grâce à une approche transnosographique, soutenant d'autant leur intérêt médico-économique [2,18,23]. Afin de s'assurer de la cohérence d'une vision de soins à long terme et d'éviter les clivages institutionnels face à ces patients, la coordination de programme de crise devrait invariablement être intégrée aux services d'urgence, comme c'est le cas pour la ville de Québec, par exemple [44].

Dans une optique d'intervention en amont de la psychothérapie, les outils issus du travail motivationnel $[17,35]$ sont utilisés dans certaines équipes spécialisées dans le traitement des troubles sévères de personnalité [16]. Cette modalité de «groupe motivationnel » est élaborée dans le but d'amener les patients présentant un TPL à explorer, sans partie pris clinique ou moral, les avantages et les inconvénients d'un changement face à des manifestions évidentes d'ambivalence au traitement (absentéisme, maintien d'une toxicomanie, désengagement, mensonge). Aucune étude n'a encore évalué l'efficacité de cette modalité de traitement, mais le seul avantage du désengorgement des places de traitements occupées par des patients non motivés est majeur.

L'offre de groupes strictement de psycho-éducation de courte durée (12 séances de fréquence hebdomadaire) est une modalité peu coûteuse et nécessitant peu d'expertise de la part des intervenants. Or, de façon surprenante, cette modalité, utilisée seule, a montré des effets directs et positifs sur les symptômes aigus du TPL [48]. En effet, la prestation d'ateliers de psycho-éducation à une cohorte de patients ayant récemment reçu un diagnostic de TPL a permis une réduction significative de l'impulsivité, une diminution des troubles relationnels, et une augmentation du fonctionnement social.

Parallèlement à ces soins directs, des programmes indirects permettent une amélioration de la qualité de vie des patients, notamment en apportant du soutien aux proches des personnes atteintes via la psycho-éducation [12]. Ces programmes soutiennent un objectif de prévention par le dépistage des familles ayant des enfants à risque, et la proposition de programmes de soins adaptés ou de limitation du plein déploiement de la pathologie limite par des attitudes structurantes. Lorsque le trouble est installé, les programmes familiaux peuvent porter sur l'information, la gestion des comportements à risque et l'amélioration des relations familiales [19,27]. À ces programmes peut s'adjoindre une psychothérapie familiale. De tels programmes, associés aux services d'urgences psychiatriques, selon des modèles de crises ou 
de DBT, permettent de prévenir une aggravation des troubles et réduire les comportements autodestructeurs répétitifs.

D'autre part, des programmes de dépistage peuvent être réalisés au niveau de la médecine scolaire (lycée) sur les éléments précurseurs. Les manifestations annonciatrices d'une instabilité fondamentale dans les différents domaines de fonctionnement psychologique avec dysrégulation émotionnelle et trouble de l'identité sont en effet généralement repérables dès l'enfance [49].

Enfin, les médecins de famille, les psychiatres ou les psychologues devraient pouvoir inclure des formations aux intervenants du réseau, des outils de dépistage et des consultations propices à des prises en charge rapides des jeunes patients présentant un TPL. Dans la ville de Québec, un tel réseau a été mis en place il y a plus de dix ans, intégrant tous ces déterminants dans la prestations de soins aux troubles sévères de personnalité. Un ingrédient essentiel a été, et est toujours le transfert des connaissances aux partenaires par les experts du traitement des troubles de personnalité. Le maintien de ces connaissances et sa mise à jour sont tout aussi importants et font souvent défaut. Cet enseignement met l'emphase sur les concepts clés dans le traitement de ces personnes : l'élaboration et le maintien d'un cadre de traitement, la gestion de la dangerosité, la compréhension du clivage et de l'identification projective, l'adoption d'attitudes responsabilisantes favorisant le développement de ces personnes.

\section{Conclusion}

Devant l'ampleur des difficultés et l'abondance des lieux d'expression des comportements des personnes présentant un TPL, il est clair que la seule modalité de traitement qu'est la psychothérapie ne peut répondre à tous les besoins. Les psychothérapeutes peuvent difficilement aider ces personnes en étant isolés d'un réseau de soin séquentiel et intégré avec toutes les lignes de soins (hospitalisation, centre de crise, services communautaires, psychothérapie, suivi médical). En effet, les alternatives à la psychothérapie passent par plusieurs voies : travail en réseau impliquant les acteurs des soins et services physiques, psychologiques et sociaux, application d'approches de psycho-éducation en groupe, traitement de la crise et approches ciblant des caractéristiques du trouble (par exemple la toxicomanie). L'objectif distal est de créer une cohérence systémique encourageant l'utilisation intégré de l'ensemble du dispositif de soins par les personnes souffrant d'un TPL : unités d'hospitalisation, hôpitaux de jour, centres de traitement, et autres lieux de soins. 
Les buts visés doivent être l'efficacité des traitements et l'accessibilité accrue à des soins pour tous patients en droit de réclamer de l'aide pour ces difficultés.

Idéalement, les services surspécialisés devraient être offerts par le bon intervenant, à la bonne personne, et au bon moment de sa démarche de changement. Des exemples d'organisation de soins intégrés pour les personnes présentant un TPL ont fait leurs preuves ailleurs dans le monde, et la France dispose du potentiel scientifique et humain pour faire de même. Souhaitons que la communauté médicale ainsi que les autres professionnels de la santé mentale sachent unir leurs forces et mettre de côté leurs différents afin d'améliorer la cohérence des soins publiques offerts aux personnes présentant un trouble sévère de personnalité.

\section{Conflit d'intérêt : aucun}




\section{Références}

[1] American Psychiatric Association: American Psychiatric Association Practice Guidelines for the Treatment of Psychiatric Disorders: Compendium 2004 Washington, American Psychiatric Association; 2004.

[2] Andreoli A, Lalive J, Garrone G. Crise et intervention de crise en psychiatrie. Paris: SIMEP; 1986.

[3] van Asselt AD, Dirksen CD, Arntz A, Severens JL. The cost of borderline personality disorder: societal cost of illness in BPD-patients. Eur Psychiatry 2007;6:354-61.

[4] Bateman A, Fonagy P. Effectiveness of partial hospitalization in the treatment of borderline personality disorder: a randomized controlled trial. Am J Psychiatry 1999;156:1563-9.

[5] Bateman AW, Fonagy P. Effectiveness of psychotherapeutic treatment of personality disorder. Br J Psychiatry 2000;177:138-43.

[6] Bateman A, Fonagy P. Treatment of borderline personality disorder with psychoanalytically oriented partial hospitalization: an 18-month follow-up. Am J Psychiatry 2001;158:36-42.

[7] Bateman A, Fonagy P. 8-Year Follow-Up of Patients Treated for Borderline Personality Disorder: Mentalization-Based Treatment Versus Treatment as Usual. Am J Psychiatry 2008;165:631-8.

[8] van Beek N, Verheul R. Motivation for treatment in patients with personality disorders. J Personal Disord 2008;22:89-100.

[9] Bellino S, Paradiso E, Bogetto F. Efficacy and tolerability of pharmacotherapies for borderline personality disorder. CNS Drugs 2008;22:671-92.

[10] Bender DS. The therapeutic alliance in the treatment of personality disorders. J Psychiatr Pract 2005;11:73-87.

[11] Bender DS, Dolan RT, Skodol AE, Sanislow CA, Dyck IR, McGlashan TH, et al. Treatment utilization by patients with personality disorders. Am J Psychiatry 2001;158:295302.

[12] Berkowitz CB, Gunderson JG. Multifamily psychoeducational treatment of borderline personality disorder. New York: Guilford; 2002.

[13] Binks CA, Fenton M, McCarthy L, Lee T, Adams CE, Duggan C. Psychological therapies for people with borderline personality disorder. Cochrane Database Syst Rev 2006:CD005652. 
[14] Blum N, Pfohl B, John DS, Monahan P, Black DW. STEPPS: A cognitive-behavioral systems-based group treatment for outpatients with borderline personality disorder-a preliminary report. Compr Psychiatry 2002;43:301-10.

[15] van den Bosch LM, Verheul R, Schippers GM, van den Brink W. Dialectical Behavior Therapy of borderline patients with and without substance use problems. Implementation and long-term effects. Addict Behav 2002;27:911-23.

[16] Bouchard S, Guilbault C, Keagan S. Alternative à la psychothérapie pour les patients avec trouble de personnalité borderline. Les groupes motivationnels. Québec ; 2008.

[17] Bouchard S, Russo J, Keagan S. Alternative à la psychothérapie pour les patients avec trouble de personnalité borderline. Les groupes motivationnels ; 2008.

[18] Burnand Y, Andreoli A, Kolatte E, Venturini A, Rosset N. Psychodynamic psychotherapy and clomipramine in the treatment of major depression. Psychiatr Serv 2002;53:585-90.

[19] Busque H. Le support direct aux enfants et aux parents qui ont un proche ayant un trouble de la personnalité. In: Gestion de crises et alternatives à la psychothérapie. Montréal, Canada; 2008.

[20] Cailhol L, Damsa C, Bui E, Klein R, Adam E, Schmitt L, et al. La recherche d'un diagnostic de trouble de personnalité borderline chez les suicidants est-elle utile aux urgences ? L'Encéphale 2008;34:23-30.

[21] Clarkin JF, Levy KN, Lenzenweger MF, Kernberg OF. Evaluating three treatments for borderline personality disorder: A multiwave study. Am J Psychiatry 2007;164:922-8.

[22] Comtois KA, Russo J, Snowden M, Srebnik D, Ries R, Roy-Byrne P. Factors associated with high use of public mental health services by persons with borderline personality disorder. Psychiatr Serv 2003;54:1149-54.

[23] Damsa C, Lazignac C, Cailhol L, Di Clemente T, Haupert J, Pull C. Major depressive disorders and psychiatric emergencies: a naturalistic study on crisis intervention relevance. Can J Psychiatry 2005;50:857-62.

[24] Fallon P. Travelling through the system: The lived experience of people with borderline personality disorder in contact with psychiatric services. J Psychiatr Ment Health Nurs 2003;10:393-401.

[25] Giesen-Bloo J, van Dyck R, Spinhoven P, van Tilburg W, Dirksen C, van Asselt T, et al. Outpatient psychotherapy for borderline personality disorder: randomized trial of schemafocused therapy vs transference-focused psychotherapy. Arch Gen Psychiatry 2006;63:64958. 
[26] Gross R, Olfson M, Gameroff M, Shea S, Feder A, Fuentes M, Lantigua R, Weissman MM. Borderline personality disorder in primary care. Arch Intern Med 2002;162:53-60.

[27] Gunderson GJ. Bordeline personality disorder. Wshington: American Psychiatric Publishing, inc; 2001.

[28] Lieb K, Zanarini MC, Schmahl C, Linehan MM, Bohus M. Borderline personality disorder. Lancet 2004;364:453-61.

[29] Linehan MM, Armstrong HE, Suarez A, Allmon D, Heard HL. Cognitive-behavioral treatment of chronically parasuicidal borderline patients. Arch Gen Psychiatry 1991;48:1060 4.

[30] Linehan MM, Heard HL, Armstrong HE. Naturalistic follow-up of a behavioral treatment for chronically parasuicidal borderline patients. Arch Gen Psychiatry 1993;50:971-4.

[31] Linehan MM, Dimeff LA, Reynolds SK, Comtois KA, Welch SS, Heagerty P, et al. Dialectical behavior therapy versus comprehensive validation therapy plus 12-step for the treatment of opioid dependent women meeting criteria for borderline personality disorder. Drug Alcohol Depend 2002;67:13-26.

[32] Linehan MM, Comtois KA, Murray AM, Brown MZ, Gallop RJ, et al. Two-year randomized controlled trial and follow-up of dialectical behavior therapy vs therapy by experts for suicidal behaviors and borderline personality disorder. Arch Gen Psychiatry 2006;63:757-66.

[33] McQuillan A, Nicastro R, Guenot F, Girard M, Lissner C, Ferrero F. Intensive dialectical behavior therapy for outpatients with borderline personality disorder who are in crisis. Psychiatr Serv 2005;56:193-7.

[34] Mehran F. Traitement du trouble de la personnalité borderline : thérapie cognitive émotionnelle, approche intégrative. Paris : Masson ; 2006.

[35] Miller WR, Rollnick S. L'entretien motivationnel : aider la personne à engager le changement. Paris : InterEditions ; 2006.

[36] Paris J. Clinical trials of treatment for personality disorders. Psychiatr Clin North Am 2008;31:517-26.

[37] Rieu J, Cailhol L, Schmitt L. Enquête de prévalence sur les troubles de personnalité en médecine générale. In: Congrès de l'Encéphale. Paris ; 2007.

[38] Rinne T, Ingenhoven T. Pharmacotherapy of severe personality disorders: a critical review. Cambridge: Cambridge University Press; 2007. 
[39] Spinhoven P, Giesen-Bloo J, van Dyck R, Kooiman K, Arntz A. The therapeutic alliance in schema-focused therapy and transference-focused psychotherapy for borderline personality disorder. J Consult Clin Psychol 2007;75:104-15.

[40] Stone MH. Clinical guidelines for psychotherapy for patients with borderline personality disorder. Psychiatr Clin North Am 2000;23:193-210.

[41] Stone DK. Personality-Disordered Patients: Treatable and Untreatable. Washington: American Psychiatric Publishing Inc; 2006.

[42] Torgersen S, Kringlen E, Cramer V. The prevalence of personality disorders in a community sample. Arch Gen Psychiatry 2001;58:590-6.

[43] Verheul R, van den Bosch LM, Koeter MW, de Ridder MA, Stijnen T, van den Brink W. Dialectical behaviour therapy for women with borderline personality disorder: 12-month, randomised clinical trial in The Netherlands. Br J Psychiatry 2003;182:135-40.

[44] Villeneuve É, Benoît A, Bouchard S. Centre de traitement Le Faubourg Saint-Jean : un travail d'équipe, un travail en réseau. Saint-Jérome : Ressources ; 2008.

[45] Weinberg I, Gunderson JG, Hennen J, Cutter CJ Jr. Manual assisted cognitive treatment for deliberate self-harm in borderline personality disorder patients. J Personal Disord 2006;20:482-92.

[46] Wilkinson SM, Gabbard GO. Management of counter-transference with borderline patients. Whashington DC: American Psychiatric Press; 1994.

[47] Zanarini MC, Frankenburg FR, Hennen J, Silk KR. Mental health service utilization by borderline personality disorder patients and Axis II comparison subjects followed prospectively for 6 years. J Clin Psychiatry 2004;65:28-36.

[48] Zanarini MC, Frankenburg FR. A preliminary, randomized trial of psychoeducation for women with borderline personality disorder. J Personal Disord 2008;22:284-90.

[49] Zelkowitz P, Paris J, Guzder J, Feldman R, Roy C, Rosval L. A five-year follow-up of patients with borderline pathology of childhood. J Personal Disord 2007;21:664-74. 